\title{
Donor-to-Acceptor Distance Dependent Fluorescence Resonance Energy Transfer Efficiency for Multiple Donors and Acceptors System Confined within 2-Dimensional Fluid of Supported Lipid Bilayer*
}

\author{
Kazuaki Furukawa ${ }^{\dagger}$ and Joana Durao ${ }^{\ddagger}$ \\ NTT Basic Research Laboratories, NTT Corporation, \\ 3-1 Morinosato Wakamiya, Atsugi, Kanagawa 243-0198, Japan \\ (Received 28 December 2011; Accepted 2 March 2012; Published 21 April 2012)
}

\begin{abstract}
We study experimentally the dependence of fluorescence resonance energy transfer (FRET) efficiency on the distance between donors and acceptors that are embedded in a supported lipid bilayer. The FRET efficiencies are determined by comparing the donor fluorescence intensities before and after acceptor photobleaching. The obtained data are compared with a theory that considers multiple donors and multiple acceptors existing in a twodimensional field. We achieve good qualitative agreement in terms of the relationship between the FRET efficiency and the donor-to-acceptor distances. The Förster radius is also estimated from the comparison, which shows good quantitative agreement. The significance of the present demonstration is in showing that acceptor photobleaching experiments can quantitatively evaluate the FRET phenomenon, which indicates that the supported lipid bilayer behaves as an ideal two-dimensional fluidic system for embedded molecules. [DOI: 10.1380/ejssnt.2012.121]
\end{abstract}

Keywords: Supported Lipid bilayer; Fluorescence Resonance Energy Transfer; Biophysics, medical physics, and biomedical engineering; Solid-liquid interfaces; Biosensing and devices

\section{INTRODUCTION}

Fluorescence resonance energy transfer (FRET) is the process whereby a molecule (called a donor) in an excited state transfers its energy nonradiatively to another kind of molecule (called an acceptor) existing in close proximity $[1,2]$. The process is due essentially to a dipole-dipole interaction. The efficiency is described by the following equation for a donor-acceptor pair.

$$
E_{F R E T}=\frac{R_{0}^{6}}{R_{0}^{6}+R^{6}}
$$

Here $R, R_{0}$ and $E_{F R E T}$ are the donor-to-acceptor (DA) distance, the Förster radius and the FRET efficiency, respectively. The Förster radius is a characteristic parameter for a donor-acceptor pair, and it gives the $\mathrm{D}-\mathrm{A}$ distance at which $E_{F R E T}$ becomes 0.5. The Förster radius is less than $10 \mathrm{~nm}$ for most donor-acceptor pairs.

On the basis of $R_{0}$, the FRET efficiency has a moderate value when $R<R_{0}$ and suddenly becomes close to zero when $R>R_{0}$. This is because the FRET efficiency is in inverse proportion to the sixth power of the $\mathrm{D}-\mathrm{A}$ distance when $R$ becomes long. In addition, the FRET observation is a noninvasive optical observation. These features make FRET a very important and useful tool for biological science. For instance it is used for observing the membrane fusion [3-6] and the interaction of proteins with other proteins and molecules [7-9]. Many kinds of dye-conjugated biomolecules are now commercially available.

In general, a sample used for FRET observation contains multiple donors and multiple acceptors all of which are involved in the FRET process. The membrane contains many lipid molecules that are always moving within

\footnotetext{
* This paper was presented at the 6th International Symposium on Surface Science - Towards Nano, Bio and Green Innovation-, Tower Hall Funabori, Tokyo, Japan, December 11-15, 2011.

†Corresponding author: furukawa.kazuaki@lab.ntt.co.jp

$\ddagger$ Present address: Institute for Molecular and Cell Biology, Rua do Campo Alegre, 823, 4150-180 Porto, Portugal
}

the membrane by lateral diffusion, and the proteins often possess multiple sites to which molecules bind. In these cases, Eq. (1), which determines the FRET efficiency for a donor-acceptor pair, is no longer applicable. It has to be modified for multiple donor and acceptor systems. The theoretical modification of Eq. (1) has already been actively studied [10-13]. However, a limited number of experimental data have been provided compared with the number of theoretical reports $[14,15]$.

When we want to observe the phenomena occurring at cell membranes, it is necessary to use a sample that maintains the fluidity of the cell membrane. A vesicle is usually used for the purpose. On the other hand, fluidity is also maintained for a supported lipid bilayer (SLB), namely a single lipid bilayer on a solid surface [16-19]. The diffusion constant determined for an SLB is close to that of vesicles [20]. This indicates that an SLB can be used instead of vesicles for measuring the properties in membranes under fluidic conditions. There are several more advantages to using an SLB. The SLB can be prepared only on a hydrophilic surface, thus it is easy to fabricate an SLB pattern by using the surface hydrophilic/hydrophobic pattern $[21,22]$. As the SLBs are adsorbed on a solid surface, they can be readily located. It is also a major advantage that we can use measurement techniques designed for solid surfaces, which are not applicable to vesicles in solution [23-25].

This paper describes the experimental determination of FRET efficiency between dyes embedded in a fluidic SLB under multiple donor-multiple acceptor conditions. The efficiency is determined from the ratios of the donor fluorescence intensities before and after acceptor photobleaching. We performed experiments using NBD (donor) and Texas Red (acceptor) in which we varied their average distances in the fluidic SLB. We compared the obtained data with a model theory for calculating the FRET efficiency of multiple donor and acceptor dyes freely mobile within two dimensions. The $\mathrm{D}-\mathrm{A}$ distance and the efficiency agree qualitatively in the model theory. We also estimated Förster radius from the observed data, which included a slight quantitative disagreement that can be 


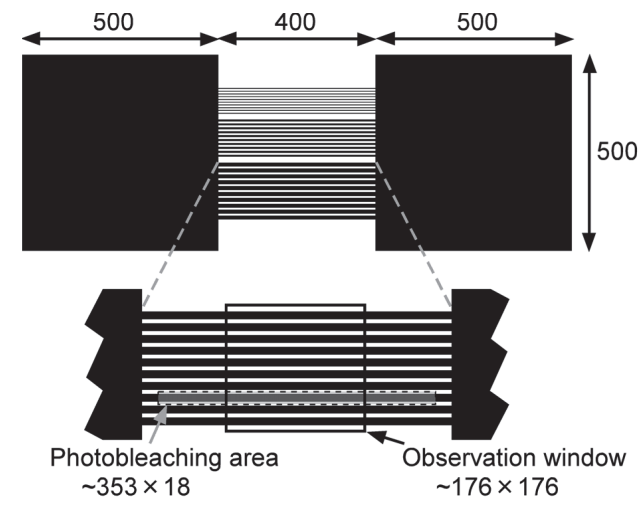

FIG. 1: The pattern used for the experiments. The numbers are in $\mu \mathrm{m}$ units.

attributed to incomplete acceptor photobleaching. The disagreement can be corrected by considering the effect of FRET induced by the unbleached acceptors. The corrected data are in better qualitative agreement with the model, and give $R_{0} \sim 4.5 \mathrm{~nm}$, namely there is good quantitative agreement with the known Förster radius between NBD and Texas Red.

\section{MATERIALS AND METHODS}

A micropattern was fabricated on a $\mathrm{SiO}_{2}$ wafer by a conventional photolithographic technique using TSMRV90 (Tokyo Ohka Kogyo) photoresist. The metal pattern (approximately $5 \mathrm{~nm} \mathrm{Ti}$ and $45 \mathrm{~nm} \mathrm{Au}$ ) was fabricated with a conventional lift-off process, and is shown in Fig. 1. It consists of three sets of 10 parallel lines with widths of 2,5 , and $10 \mu \mathrm{m}$. We used a set of $10 \mu \mathrm{m}$ wide lines in the present study.

L- $\alpha$-phosphatidylcholine (L- $\alpha$-PC) extracted from egg yolk was purchased from Sigma-Aldrich. The dyeconjugated lipids NBD-DHPE and Texas Red-DHPE were purchased from Avanti Polar Lipid Inc. and Invitrogen, respectively. Chloroform and sodium chloride were purchased from Kanto Chemical Co. Inc. A $1 \mathrm{~mol} / \mathrm{L}$ Tris-HCl buffer $(\mathrm{pH}=7.6)$ was purchased from Nacalai Tesque. Deionized water (Millipore, >18 $\mathrm{M} \Omega \cdot \mathrm{cm}$ ) was used throughout the work.

The samples were prepared according to our previously reported method $[26,27]$. A 1:1 mixture of NBD-DHPE and Texas Red-DHPE was first prepared as a chloroform solution, which was then diluted to various concentrations with L- $\alpha$-PC. The solution was dried in a nitrogen stream to yield a sticky solid. A small amount of the solid was spotted on the inside surface of two $500 \mu \mathrm{m}$ square wells. The sample was immersed in buffer solution consisting of $100 \mathrm{mM} \mathrm{NaCl}$ and $10 \mathrm{mM}$ Tris- $\mathrm{HCl}(\mathrm{pH}=7.6)$ to initiate self-spreading. After a certain period of time, the self-spreading SLBs collide with each other in the middle of the parallel lines. After confirming the collision, we left the sample for at least $1 \mathrm{~h}$ to obtain a uniform distribution of the dye-conjugated lipid molecules in the formed SLB. This is required because it is known that dye-conjugated lipid molecules often have a concentration distribution during self-spreading $[28,29]$.

The acceptor photobleaching experiments were performed as follows. The photobleaching area was double the length the observation area that consisted of lines approximately $180 \mu \mathrm{m}$ long (Fig. 1). We took a sufficient area for photobleaching to avoid the effects of acceptor molecules carried by lateral diffusion on the fluorescence recovery after photobleaching in the observation area. The fluorescence images were recorded every $1 \mathrm{~min}$ of photobleaching time for the first $10 \mathrm{~min}$. Then the final image was taken after 10 more min of photobleaching (total bleaching time was $20 \mathrm{~min}$ ).

An Olympus BX51-FV300 confocal laser-scanning microscope equipped with $488 \mathrm{~nm}(100 \mathrm{~mW})$ and $543 \mathrm{~nm}(5$ $\mathrm{mW}$ ) laser light sources for excitation was used for observing the images. We used a 505-525 nm filter with a $488 \mathrm{~nm}$ light source for the NBD fluorescence observations and a $610 \mathrm{~nm}$ high-pass filter with a $543 \mathrm{~nm}$ light source for the Texas Red observations. For the acceptor photobleaching, we used the maximum power of the $543 \mathrm{~nm}$ laser. However, we chose the minimum excitation laser power (less than $1 \%$ of the full power of our system) and set the photomultiplier voltage and gain within a range that avoided saturating the output signals so that the photomultiplier output can be considered to have a fluorescence intensity that is proportional to the number of dye molecules in the observation area. A water-immersion objective lens (Plan Apo 40×WLSM (Olympus)) was used for all the observations. The space between the sample and the objective lens was filled with buffer solution throughout the observations. All observations were carried out at room temperature.

\section{RESULTS AND DISCUSSION}

\section{A. Donor fluorescence recovery after acceptor photobleaching}

Figure 2 shows fluorescence images recorded with an LSM at various photobleaching times. It shows two lines for each photobleaching time, which indicates an SLB without (top) and with (bottom) photobleaching. Since the SLB is confined within the lines, we can photobleach only one of the lines. The initial conditions before photobleaching (indicated as $0 \mathrm{~min}$ ) are FRET conditions. The green fluorescence intensity is from donors (NBD) that escaped the FRET process. Thus, as the acceptor photobleaching progresses, the red fluorescence from the acceptor, Texas Red, is weakened while the green fluorescence is recovered.

Figure 2(a) is a combined image of red and green $\mathrm{flu}-$ orescence. It is clear from the bottom lines at each photobleaching time that the green fluorescence intensities increased as the photobleaching time increased. To compare the changes of donor and acceptor fluorescence more clearly, Figs. 2(b) and (c) show the changes in the fluorescence intensities of the donor and acceptor separately. In Fig. 2(b), the donor fluorescence intensity is recovered on the acceptor photobleaching line. This result indicates that the initial condition was certainly in the FRET conditions. Correspondingly, the acceptor fluorescence intensity becomes weaker as the photobleaching time increases 

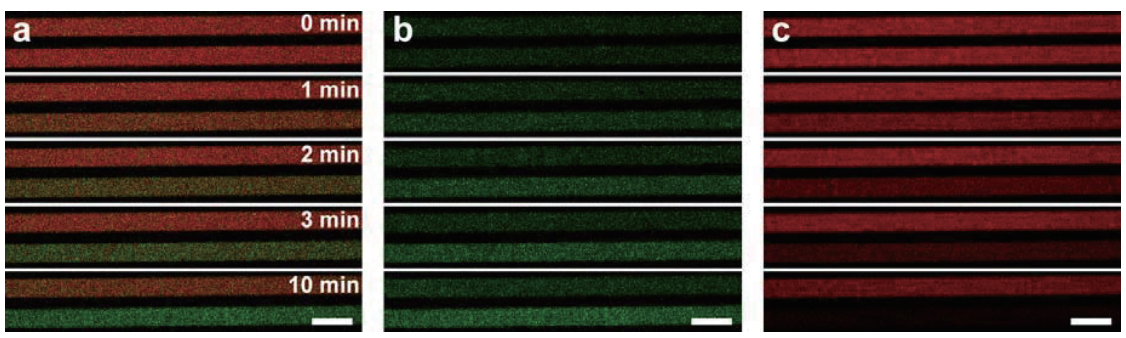

FIG. 2: Confocal laser scanning microscope images of SLB containing 1\% of NBD-DHPE and Texas Red-DHPE 1:1 mixture, depending on acceptor photobleaching time. The top line in each image is a reference and the bottom line is used for acceptor photobleaching. Scale bar: $20 \mu \mathrm{m}$. (a) Combined image of (b) and (c). (b) Fluorescence between 505 and $525 \mathrm{~nm}$ realized by $488 \mathrm{~nm}$ excitation. (c) Fluorescence over $610 \mathrm{~nm}$ realized by $543 \mathrm{~nm}$ excitation.

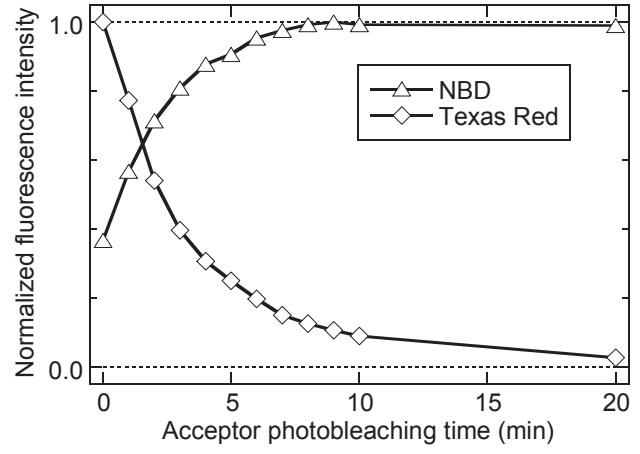

FIG. 3: Fluorescence intensities from an SLB containing $1 \%$ of a Texas Red (acceptor) and NBD (donor) 1:1 mixture as a function of acceptor photobleaching time. The intensities are normalized by the maximum intensities observed for each.

in Fig. 2(c). The acceptor photobleaching time of $1 \mathrm{~min}$ is not effective and a lot of acceptor fluorescence was observed. After $10 \mathrm{~min}$, the acceptor fluorescence became very weak, but had not perfectly disappeared.

We would like to emphasize here that experiments performed using different lines fabricated on the same substrate can be considered different experiments using independent samples. Thus we can perform an acceptor photobleaching experiment using the bottom line while retaining the initial condition in the top line in Fig. 2. This gives us a significant advantage when we compare our experiments with the others using samples prepared independently. With the samples we used, we could minimize the difference between samples used for observation and for control experiments owing to the preparation conditions.

\section{B. FRET efficiency estimated by donor fluorescence recovery after acceptor photobleaching}

Figure 3 plots the relative fluorescence intensities from donors and acceptors versus the acceptor photobleaching time for a sample containing $1 \%$ of the $1: 1$ mixture of NBD-DHPE and Texas Red-DHPE. This corresponds that the sample contains $0.5 \%$ of donors and acceptors each. The fluorescence intensities are averaged for the
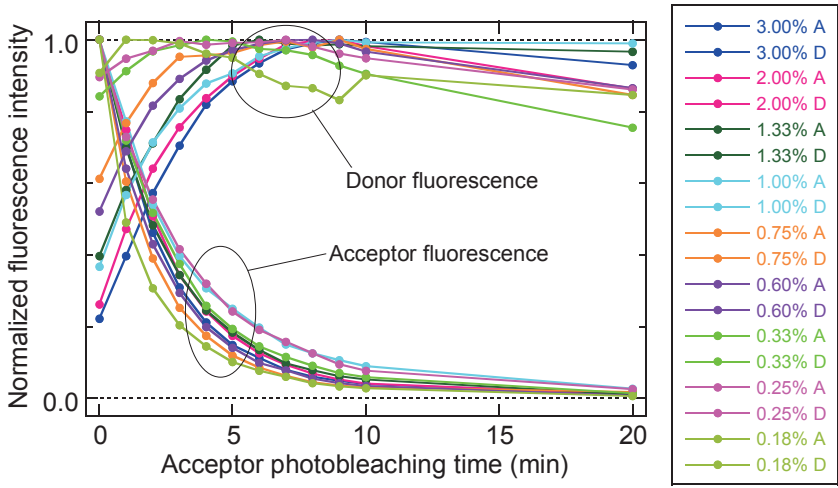

FIG. 4: Fluorescence intensities from an SLB containing a Texas Red (acceptor) and NBD (donor) 1:1 mixture with various concentrations as a function of acceptor photobleaching time. The intensities are normalized by the maximum intensities observed for each.

entire observation area within the line in Fig. 2. The intensities are normalized by the maximum value of the photodetector output for the donors and acceptors. It is quite natural for the acceptor to have a maximum value of 1 under the initial conditions. As the acceptor fluorescence is weakened by photobleaching, the donor fluorescence recovers. Its maximum appears after 9 min of photobleaching (thus the value at $9 \mathrm{~min}$ is normalized as 1). Again, the observation of donor fluorescence recovery provides solid proof that this system was under FRET conditions before photobleaching. Although an increase in the photobleaching time deactivates more acceptors, about $3 \%$ of the acceptor fluorescence still remains after 20 min of bleaching. At the same time the donor fluorescence becomes weaker. This is because the laser used for the acceptor bleaching also slightly degrades the donors. The observation every minute also affects donor degradation because it uses the excitation wavelength for the donors. However, we believe that the latter degradation is limited by using a very small excitation power (less than $1 \%$ of the maximum power) for the observations. This is supported by the images in Fig. 2(b) that show the almost constant donor fluorescence from the reference line.

For the preliminary analyses in the present study, we assume that the difference in the fluorescence intensities 


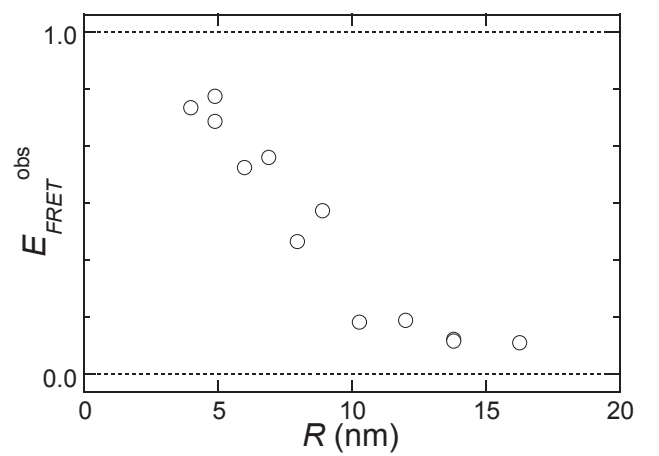

FIG. 5: Plot of $E_{F R E T}^{\mathrm{obs}}$ determined as a function of an average D-A distance $R$.

before and after acceptor photobleaching is related to the difference of the system in FRET and no FRET conditions. Then the FRET efficiency can be estimated from the changes in the donor fluorescence intensities. Since the fluorescence intensities are normalized by the maximum recovery, the difference between 1 and the initial donor fluorescence intensity $F_{\text {init }}$ can be regarded as the FRET efficiency determined by the observations, $E_{F R E T}^{\text {obs }}$, as

$$
E_{F R E T}^{\mathrm{obs}}=1-F_{\text {init }} .
$$

Thus we proceed to the following discussions based on the values estimated with Eq. (2). We have to be careful to ensure that $E_{F R E T}^{\mathrm{obs}}$ is always smaller than the real values. This is because the acceptor photobleaching can never be perfect. However, it is still worth using these efficiencies for an initial discussion of the dependence of the change in the FRET efficiency on the average $\mathrm{D}-\mathrm{A}$ distance.

\section{FRET efficiency dependence on D-A distance}

Using the method described above, we next performed experiments employing samples with various DA distances in a two-dimensional membrane. This was achieved by changing the concentration of the 1:1 mixture of NBD and Texas Red in L- $\alpha$-PC. The dependence of the donor and acceptor fluorescence intensities on the acceptor photobleaching time is plotted in Fig. 4. They showed a similar tendency to those in Fig. 3. The donor fluorescence recovery becomes efficient when the acceptor is photobleached, and reaches its maximum value at a certain bleaching time, which differs depending on the concentration. In general, samples with a low concentration reach their maximum value in a shorter photobleaching time than those with high concentrations.

The FRET efficiency can be estimated from the normalized initial donor fluorescence intensities. The estimated $E_{F R E T}^{\text {obs }}$ values are plotted against the average D-A distances in Fig. 5. Here, the average distance was set to 6.8 $\mathrm{nm}$ for the $1 \%$ sample, which was derived from the knowledge that $1 \mu \mathrm{m}^{2}$ lipid bilayer contains about $5 \times 10^{6}$ lipid molecules in a common animal cell $[21,30]$. It is clear that $E_{F R E T}^{\text {obs }}$ decreases as the average D-A distance increases. The decrease is not proportional to the $\mathrm{D}-\mathrm{A}$ distance and

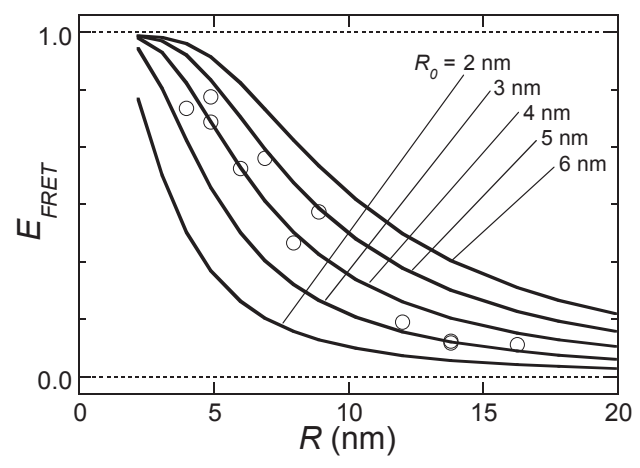

FIG. 6: FRET efficiencies calculated with various $R_{0}$ values using Eq. (3) and (4). The circles are $E_{F R E T}^{\text {obs }}$ determined in Figure 5.

the efficiency becomes almost constant at a very small value when the $\mathrm{D}-\mathrm{A}$ distance is more than $10 \mathrm{~nm}$. This corresponds to the inverse proportion of $E_{F R E T}$ to the sixth power of the $\mathrm{D}-\mathrm{A}$ distance in the long $R$ region in Eq. (1).

\section{Förster radius estimated by comparison with a theoretical model}

Next we estimate the Förster radius from our present experimental data. For this purpose, we require a model that determines the FRET efficiency between donors and acceptors in two dimensions, rather than Eq. (1). Several calculation methods have been reported for this purpose. Of these models, we use a simple one that assumes a random distribution of donors and acceptors, and does not consider the dynamics of excited states and the volume exclusion of the dyes when analyzing our present data $[2,10]$. The equation is

$$
I_{D A}(t)=I_{D}^{0} \exp \left[-\frac{t}{\tau_{D}}-2 \beta\left(\frac{t}{\tau_{D}}\right)^{\frac{1}{3}}\right],
$$

where

$$
\beta=\frac{\Gamma(2 / 3)}{2} \frac{C}{C_{0}}, C_{0}=\frac{1}{\pi R_{0}^{2}} .
$$

Here, $I_{D A}(t)$ and $I_{D}^{0}$ are the fluorescence intensities from donors in the presence/absence of acceptors. The parameters $C_{0}, C$ and $\tau_{D}$ are the area occupied by the circle of the Förster radius, the area occupied by an acceptor, and the donor lifetime without acceptors, respectively. The FRET efficiency corresponds to the integral of $I_{D A}(t) / I_{D}^{0}$ from $t=0$ to infinity. As in Eq. (4), the acceptor concentration is a parameter for the calculation. We calculated $E_{F R E T}$ against the average $\mathrm{D}-\mathrm{A}$ distance for various $R_{0}$ values as shown in Fig. 6 together with the data plotted in Fig. 5. It should be noted that $1 \%$ in Fig. 3 means the acceptor concentration of $0.5 \%$ in the present notation. The FRET efficiency in Fig. 4 is larger than that calculated using Eq. (1) for the same $R_{0}$. This is due to the fluidity assumed in the model represented by Eq. (3). 


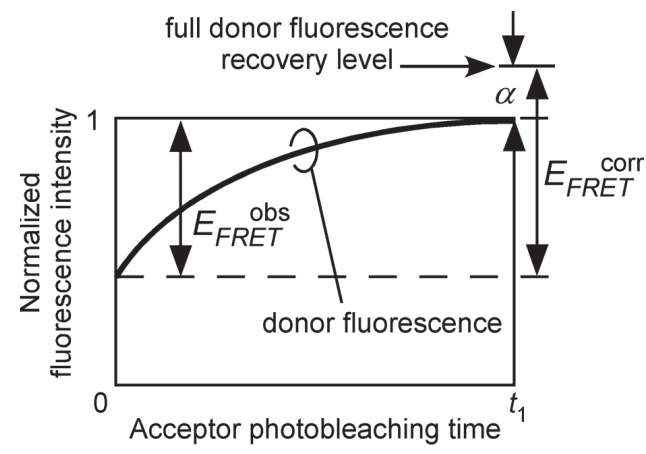

FIG. 7: Schematic diagram of the relation between $E_{F R E T}^{\text {obs }}$ and $E_{F R E T}^{\text {corr }}$ for determining the corrected FRET efficiencies considering the acceptor molecules that remained unbleached.

In Fig. 6, $E_{F R E T}^{\mathrm{obs}}$ tends to follow the theoretical calculations. This implies that the FRET efficiencies that depend on $\mathrm{D}-\mathrm{A}$ distance are properly determined with our experimental setup, and that the simulation of the FRET efficiencies using the simple theory is effective.

The experimental data seem to be best fit by the calculated value of $4 \mathrm{~nm}$ in Fig. 6, although the data include small discrepancies. The reported $R_{0}$ is $\sim 5.0 \mathrm{~nm}$ for a NBD-Texas Red pair [31]. Our recent experiments using a self-spreading supported lipid bilayer concluded a Förster radius of $5.3 \mathrm{~nm}$ for the pair [32]. When we compare the radius of about $4 \mathrm{~nm}$ determined here with these previously reported data, the Förster radius provided by the acceptor photobleaching technique is short. To find a reasonable explanation for this discrepancy, we further inspect our experiments and their interpretations.

\section{E. Corrected FRET efficiency considering effect of leaving acceptor unbleached}

In the above discussion, we attempted to determine $E_{F R E T}^{\text {obs }}$ by comparing the donor fluorescence intensities before and after acceptor photobleaching. However, as indicated in Figs. 3 and 4, the fluorescence from acceptor molecules does not disappear completely in the observation area after a certain period of photobleaching.

We think two main possibilities should be discussed; one is the inflow of unbleached acceptors caused by the lateral diffusion of the SLB and the other is the unavoidable acceptor residue of photobleaching experiments. We focused on the possible inflow at an early stage of this work, and we set the photobleaching area more than the observation area as shown in Fig. 1. The possible inflow of acceptor molecules from the unbleached area to the observation area can be estimated by the solution of the diffusion equation,

$$
C(x, t)=\frac{1}{\sqrt{4 \pi D t}} \exp \left[\frac{-x^{2}}{4 D t}\right]
$$

where $C(x, t), D, t$, and $x$ are the concentration of diffusing molecules, diffusion constant, time and distance, respectively. In the current measurements, observation (2.7 s) and photobleaching (1 min) are repeated 10 times.
The inflow of the unbleached acceptor molecules into the observation area can be calculated with Eq. (5). Taking the initial condition by distributing dye-conjugated lipid molecules homogeneously in the region at $x \leq 0$ and none at $x>0$ at $t=0$, the calculated inflow quantity is almost zero, if we assume that the diffusion constant $D$ is $10 \mu \mathrm{m}^{2} \mathrm{~s}^{-1}$, which is a relatively large value for an SLB. This negligible inflow quantity is due to the long distance between the unbleached edge and the observation edge $(x$ $=88 \mu \mathrm{m})$ after a short period of time $(t=2.7 \mathrm{~s})$ relative to the diffusion constant.

Therefore, the incomplete acceptor photobleaching is most likely due to the practical problem intrinsic to acceptor photobleaching experiments. About 0.78 of acceptor molecules are bleached by 1 min photobleaching as seen in Fig. 3. For instance, if the bleaching is repeated 10 times, we calculate that $(0.78)^{10} \sim 0.08$ acceptor molecules will be left unbleached. The calculated value is in good agreement with the observed fluorescence intensity at $10 \mathrm{~min}$ shown in Fig. 3. We therefore attribute the incomplete acceptor photobleaching to the latter reason.

Since photobleaching proceeds at a constant rate to an irradiation time, the unbleached acceptor molecules can never become zero. Thus $E_{F R E T}^{\text {obs }}$ obtained in the above discussion must be corrected considering the FRET caused by the remaining acceptors. We corrected the efficiency according to the scheme shown in Fig. 7. We determined $E_{F R E T}^{\text {obs }}$ from the difference between the initial fluorescence intensity and the maximum fluorescence intensity of the donor under normalized conditions. However, the maximum donor fluorescence recovery must be more than 1. The increment is denoted as $\alpha$ in Fig. 7 . From the acceptor fluorescence intensity at $t=t_{1}$, we can calculate the FRET efficiency using Eq. (3). The calculated efficiency corresponds to $\alpha /(1+\alpha)$. By using the value of $\alpha$, the corrected FRET efficiency, $E_{F R E T}^{c o r r}$, can be calculated by $\left(E_{F R E T}^{\text {obs }}+\alpha\right) /(1+\alpha)$.

The result in Fig. 3 is a good example for examining the above calculation method. The acceptor fluorescence intensity after $1 \mathrm{~min}$ photobleaching is 0.78 . If the acceptors are bleached at this rate, the unbleached acceptors are calculated as $0.78^{9}=0.10$ at $t=9 \mathrm{~min}$, the time at which the donor fluorescence recovery becomes maximum. The observation datum at that time is 0.11 , which is in good agreement with the calculation. This means that 0.11 of the initial acceptor is left unbleached. The expected FRET efficiency under such conditions can be calculated by substituting the concentration for $C$ in Eq. (4). Here, if we assume $R_{0}=4.0 \mathrm{~nm}$, the calculated FRET efficiency is 0.077 . Therefore, the full donor fluorescence recovery is expected to be $1 /(1-0.077)=1.08$ of the donor fluorescence at $t=9 \mathrm{~min}$. The corrected FRET efficiency then becomes $E_{F R E T}^{c o r r}=0.66$, while $E_{F R E T}^{\text {obs }}=$ 0.63 . It should be noted that $E_{F R E T}^{c o r r}$ varies depending on $R_{0}$.

The corrected values of $E_{F R E T}^{\text {corr }}$ are plotted in Fig. 8 together with the theoretical calculations. Since $R_{0}$ is a parameter for the determining $E_{F R E T}^{\mathrm{corr}}$, Fig. 8 shows the results independently corrected by assuming $R_{0}=4.0$, 4.5, $5.0 \mathrm{~nm}$. Figure 8 shows better qualitative agreement between the experiments and theory. Of the three $R_{0}$ values, $R_{0}=4.5 \mathrm{~nm}$ best fits the experiments and theory. The value of $4.5 \mathrm{~nm}$ is close to the reported Förster radius 

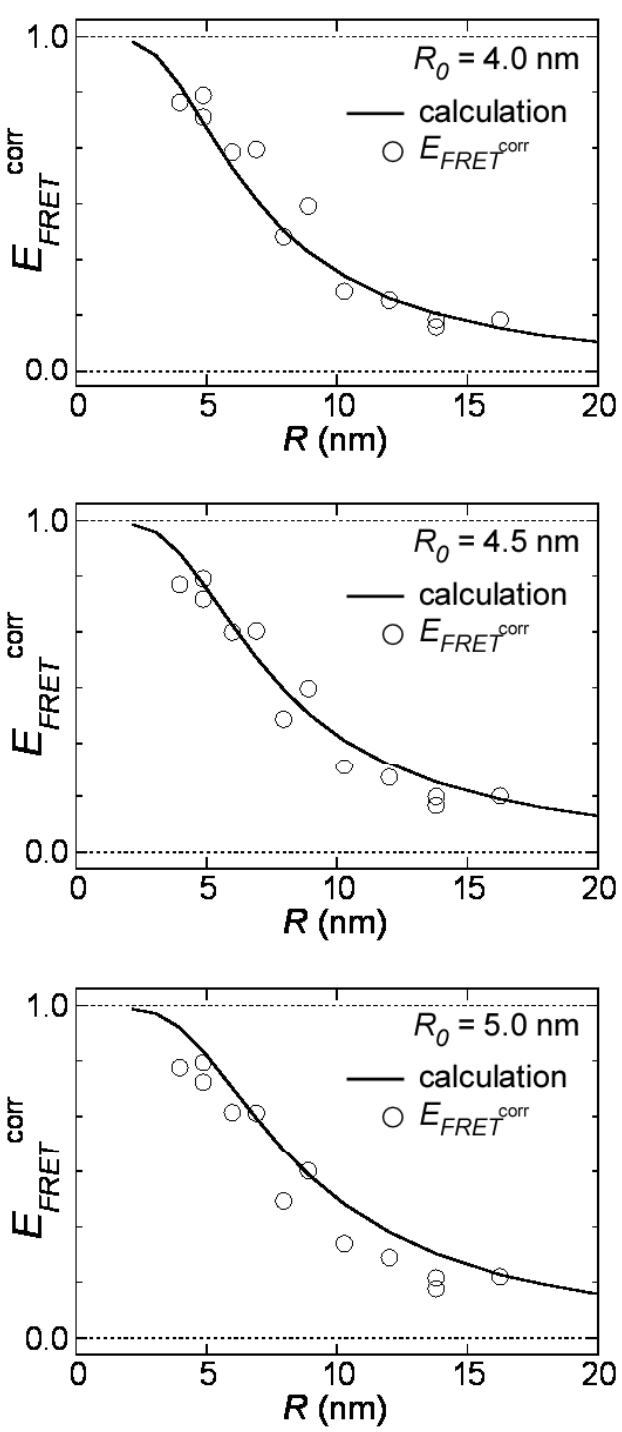

FIG. 8: Plot of corrected FRET efficiencies together with the theoretical calculation by assuming $R_{0}=4.0,4.5$ and $5.0 \mathrm{~nm}$.

between NBD and Texas Red of $5 \mathrm{~nm}$ [31].

The result in Fig. 8 indicates that the experiments and theory are in good qualitative agreement and also in quantitative agreement. This shows the validity of the correction based on Eq. (3), which in turn is based on the donor and acceptor being freely mobile and randomly distributed within the two-dimensional system. Although the model does not include further possible effects such as the interaction between donors and the dye molecule orientation effect, their contributions should be limited in the present experiments. The model also has an important implication as regards the characteristics of the sample used. The SLB offers a good model cell membrane that realizes the conditions for freely mobile molecules with a certain speed within two dimensions. This is in contrast to cases where immobilized samples are used on a solid surface [14].

The above discussion is based on the average $\mathrm{D}-\mathrm{A}$ distance of $6.8 \mathrm{~nm}$ for the $1 \%$ sample which is derived from the lipid density known for a common animal cell. The distance, however, depends on the kind of phospho-

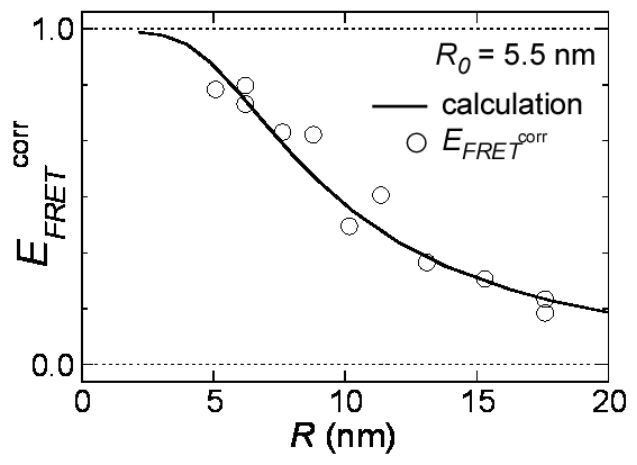

FIG. 9: Plot of corrected FRET efficiencies using a low lipid density for L- $\alpha$-PC of $3 \times 10^{6}$ lipid molecules $/ \mu \mathrm{m}^{2}$ together with the theoretical calculation by assuming $R_{0}=5.5 \mathrm{~nm}$.

lipids. It has been reported that dioleoylphosphatidylcholine (DOPC), the main composite of L- $\alpha$-PC, under the liquid crystalline phase is less dense $\left(\sim 3 \times 10^{6}\right.$ lipid molecules $\left./ \mu \mathrm{m}^{2}\right)$ than the animal cell $\left(\sim 5 \times 10^{6}\right.$ lipid molecules $/ \mu \mathrm{m}^{2}$ ) [33]. When we assume the density of DOPC for L- $\alpha$-PC, the average D-A distance becomes ca. $30 \%$ longer. We have thus compared the experimental data with the calculations under the assumption of the average D-A distance of $8.8 \mathrm{~nm}$ (ca. 30\% longer than 6.8 $\mathrm{nm}$ ) for the $1 \%$ sample. In this case, the calculations best fit the experimental data when $R_{0}=5.5 \mathrm{~nm}$ as shown in Figure 9. The value is somewhat longer than the previous reports, but is in a reasonable range for the Förster radius of the dye pair. Although the lipid density of the self-spreading SLB has not been measured, and certainly is one of the open questions for self-spreading SLB [29], the present results may give a hint to approach the problem.

Our attempt to determine the Förster radius by acceptor photobleaching experiments might be somewhat imprudent, because it includes an intrinsic disadvantage, namely that complete bleaching is almost impossible. However, as we have demonstrated here, it yielded a satisfactory result in determining the Förster radius if we set up appropriate conditions: the SLB sample and the calculation method based on the random distribution of dyes is one of the best conceivable combinations.

\section{CONCLUSIONS}

We have studied experimentally the dependence of the fluorescence resonance energy transfer (FRET) efficiency on the distance between donors and acceptors embedded within a supported lipid bilayer. The FRET efficiencies determined by comparing the donor fluorescence intensities before and after acceptor photobleaching have shown good qualitative agreement in terms of the relationship between the FRET efficiency and the donor-to-acceptor distances. Based on the theory that considers the multiple donors and multiple acceptors existing in a twodimensional field, the obtained data were corrected and compared with the theoretical calculation results. The corrected data are in good quantitative agreement with 
already reported values. We conclude that the present results can be attributed to the excellent two-dimensional fluidic property of the supported lipid bilayer. The bilayer thus can be used not only as a good model for a cell membrane but also as an ideal two-dimensional field for embedded molecules.

\section{Acknowledgments}

J.D. acknowledges internship support from Vulcanus in Japan organized by the EU-Japan Center for Industrial Cooperation.
[1] Th. Förster, Ann. Phys. 2, 55 (1948).

[2] J. R. Lakowicz, Principles of Fluorescence Spectroscopy, 3rd ed. (Springer, New York, 2006).

[3] T. N. Estep and T. E.Thompson, Biophys. J. 26, 195 (1979).

[4] D. K. Struck, D. Hoekstra, and R. E. Pagano, Biochem. 20, 4093 (1981).

[5] N. Tamai, T. Yamazaki, I. Yamazaki, A. Mizuma, and N. Mataga, J. Phys. Chem. 91, 3503 (1987).

[6] L. M. S. Loura, A. Fedorov, and M. Prieto, Biophys. J. 71, 1823 (1996).

[7] C. G. Dosremedios and P. D. J. Moens, J. Struct. Biol. 115, 175 (1995)

[8] A. Miyawaki, Dev. Cell. 4, 295 (2003).

[9] T. Ueno, H. Taguchi, H. Tadakuma, M. Yoshida, and T. Funatsu, Mol. Cell 14, 423 (2004).

[10] P. K. Wolber and B. S.Hudson, Biophys. J. 28, 197 (1979).

[11] D. E. Koppel, P. J. Fleming, and P. Strittmatter, Biochem. 18, 5450 (1979).

[12] B. K.-K. Fung and L. Stryer, Biochem. 17, 5241 (1978).

[13] J. Kusba, L. Li, I. Gryczynski, G. Piszczek, M. Johnson, and J. R. Lakowicz, Biophys. J. 82, 1358 (2002).

[14] C. Berney and G. Danuser, Biophys. J. 84, 3992 (2003).

[15] T. Zal and N. R. J. Gascoigne, Biophys. J. 86, 3923 (2004).

[16] E. Sackmann, Science 271, 43 (1996).

[17] B. Kasemo, Surf. Sci. 500, 656 (2002).

[18] M. Tanaka and E. Sackmann, Nature 437, 656 (2005).

[19] R. Richter, R. Bérat, and A. R. Brisson, Langmuir 22, 3497 (2006).
[20] J. Salafsky, J. T. Groves, and S. G. Boxer, Biochem. 35, 14773 (1996)

[21] J. T. Groves and S. G. Boxer, Acc. Chem. Res. 35, 149 (2002).

[22] L. A. Kung, J. T. Groves, N. Ulman, and S. G. Boxer, Adv. Mater. 12, 731 (2000).

[23] I. Zawisza, G. Wittstock, R. Boukherroub, and S. Szunerits Langmuir 24, 3922 (2008).

[24] Y. Shinozaki, A. M. Siitonen, K. Sumitomo, K. Furukawa, and K. Torimitsu, Jpn. J. Appl. Phys. 47, 6164 (2008).

[25] B. Takimoto, H. Nabika, and K. Murakoshi, J. Phys. Chem. C 113, 3127 (2009).

[26] K. Furukawa, H. Nakashima, Y. Kashimura, and K. Torimitsu, Lab Chip 6, 1001 (2006).

[27] K. Furukawa, K. Sumitomo, H. Nakashima, Y. Kashimura, and K. Torimitsu, Langmuir 23, 367 (2007).

[28] J. Rädler, H. Strey, and E. Sackmann, Langmuir 11, 4539 (1995).

[29] J. Nissen, S. Gritsch, G. Wiegand, and J. O. Rädler, Eur. Phys. J. B 10, 335 (1999).

[30] B. Alberts, D. Bray, J. Lewis, M. Raff, K. Roberts, and J. D. Watson, Molecular Biology of the Cell, 2nd ed. (Garland Publishing Inc., New York, 1990), Ch. 6.

[31] Y. Kaizuka, and J. T. Groves, Biophys. J. 86, 905 (2004).

[32] K. Furukawa, H. Nakashima, Y. Kashimura, and K. Torimitsu, Langmuir 24, 921 (2008).

[33] J. F. Nagle and S. Tristram-Nagle, Biochimica et Biophysica Acta 1469, 159 (2000). 